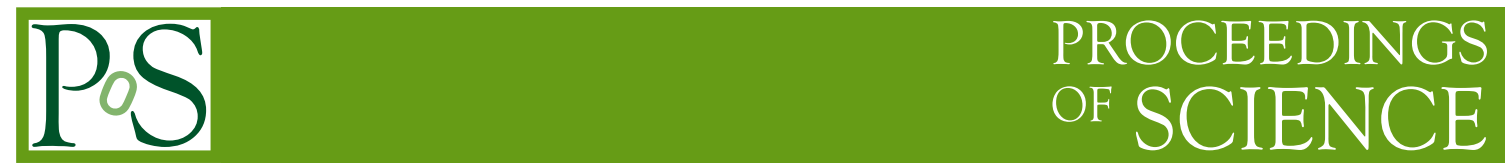

\title{
VBF SM Higgs boson searches with ATLAS
}

\section{Stefania Xella (for the ATLAS collaboration)*}

Niels Bohr Institute, Copenhagen University, Denmark

E-mail: xella@nbi.dk

The observation of a Standard Model Higgs boson produced through the Vector Boson Fusion process and its decay into tau pairs with the ATLAS detector at the Large Hadron Collider is reported. Trigger issues and impact of event pileup are also considered. The discovery potential in the lepton-lepton and lepton-hadron final states is discussed. The studies are based on the analysis of Monte Carlo signal and data background simulated in detail through the experimental apparatus.

2008 Physics at LHC

September 29 - 4 October 2008

Split, Croatia

${ }^{*}$ Speaker. 


\section{Introduction}

The search for the Higgs boson and the source of electroweak symmetry breaking is a primary task of the Large Hadron Collider (LHC). The ATLAS detector is capable of discovering the Standard Model Higgs boson with masses ranging from the LEP limit of $114 \mathrm{GeV}$ to about $1 \mathrm{TeV}$ [1]. The low-mass region is preferred from electroweak precision measurements and in this region $\left(m_{H}<130 \mathrm{GeV}\right)$ the searches for Higgs bosons decaying to taus and photons are the most promising for discovery [2]. Searches for the Higgs boson produced in Vector Boson Fusion (VBF) tend to have reasonably high signal-to-background ratios, making them more robust to systematic uncertainties. Within the Standard Model, the ability to observe the Higgs boson in multiple production and decay configurations makes it possible to measure the Higgs boson coupling to fermions and vector bosons [3]. Furthermore, the VBF processes provide a tool for measuring the Higgs boson spin and CP properties [4]. In the context of the MSSM, the branching ratio of a Higgs boson decaying to photons is generally suppressed, which makes the search for Higgs boson decaying to taus very important. The complementarity of the coupling of the light and heavy CP-even, neutral Higgs bosons of the MSSM to taus makes it possible to cover most or all of the $m_{A}-\tan \beta$ plane by reinterpreting the results for a Standard Model Higgs boson decaying into taus in the context of the MSSM [5]. In this note we have considered three decay modes: the lepton-lepton (ll-channel), lepton-hadron (lh-channel) and the hadron-hadron ( $h h$-channel) from VBF $H \rightarrow \tau^{+} \tau^{-}$signature. The analysis has been done using state-of-the art Monte Carlo generators, full GEANT-based simulation of the ATLAS detector with realistic misalignments and distortions applied to the expected material in the detector, utilization of our current reconstruction algorithms, and, where possible, incorporation of pileup interactions. The results of this study are summarized in greater details in [1].

\section{Analysis strategy}

The signal process under consideration, $\operatorname{VBF} H \rightarrow \tau^{+} \tau^{-}$, has a relatively modest cross section, of the order of $300 \mathrm{fb}$. The main backgrounds to this process are: double tau final states from $W$ or $Z$ boson production with jets of $p_{T}>20 \mathrm{GeV}(\sigma \approx 100$ 's pb), production of QCD multijets with $p_{T}>17 \mathrm{GeV}(\sigma \approx \mathrm{mb})$, top anti-top production with at least one top quark decaying into leptons $(\sigma \approx n b)$. The jet activity of the vector boson fusion process is unique in several ways, providing many handles to suppress backgrounds and isolate a sample of signal events with high purity. The most important feature of the VBF process is the presence of two high- $p_{T}$ quark-initiated "tagging jets", which tend to be relatively forward and well separated in rapidity. Furthermore, due to color coherence in this electroweak process, additional QCD radiation between the tagging jets tends to be suppressed and motivates a Central Jet Veto (CJV). This is shown in Fig. 1. An additional handle to reject backgrounds is the requirement of an identified electron, muon and tau lepton, which allows to reject a large fraction of quark or gluon initiated jets.

Although there are several neutrinos in the event, it is possible to reconstruct the $\tau^{+} \tau^{-}$invariant mass by making the approximation that the decay products of the $\tau$ are collinear with the $\tau$ in the laboratory frame ${ }^{1}$, and restricting to tau leptons decays products not back-to-back. We

\footnotetext{
${ }^{1}$ This is a good approximation since $m_{H} / 2 \gg m_{\tau}$ and hence the taus are highly boosted
} 
call this the "collinear mass", and this is the distribution used for the discovery sensitivity reported here.
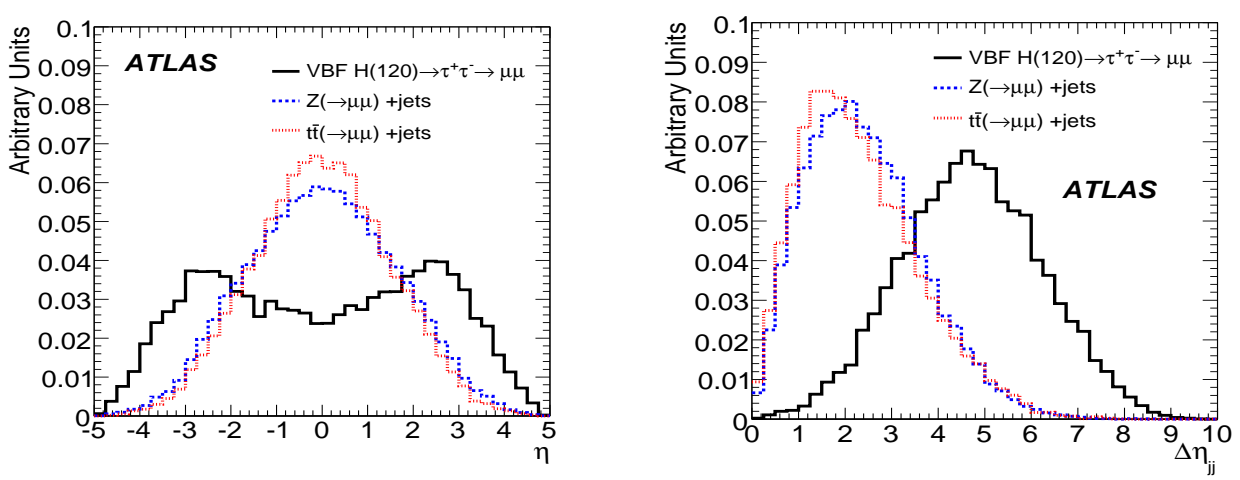

Figure 1: Pseudorapidity of the highest $p_{T}$ jet and pseudorapidity gap between tag jets in VBF $H \rightarrow \tau \tau \rightarrow$ $\mu \mu$ events and relevant backgrounds.

\section{Results}

For a Higgs mass of $120 \mathrm{GeV}$, the numbers of events expected in the collinear mass window per $f b^{-1}$ of integrated luminosity in the three channels are the following. For the $l l$-channel, 0.45 signal events are expected, with a total background of about 0.44 events (most dominant background is $Z$ boson plus jets production, followed by top anti-top production). For the $l h$-channel, 0.61 signal events are expected, with a total background of about 0.20 events (most dominant background is $Z$ boson plus jets production). For the $h h$-channel, 0.34 signal events are expected, with a total background of about 1 event $^{2}$ (most dominant background is QCD multijets production, followed by $Z$ or $W$ boson plus jets production).

In the $p_{T}$ regime relevant for the VBF SM Higgs production search, hadronic tau decays are more difficult to identify with respect to electron or muon tau decays. Therefore the $l l$ and $l h$ channels provide a more significant observation of Higgs production than the $h h$-channel. On the other hand, the study reported in [1] shows that the trigger for the $h h$-channel is feasible, and the reconstruction of the signal maintains an efficiency and $m_{\tau \tau}$ mass resolution comparable to the other channels (see Fig.2 (a-c)). The open question for this channel is the size of the QCD background, as mentioned before. This question can only be answered with data, therefore no significance for observation in the $h h$-channel is reported here.

\section{Expected significance}

The expected significance of discovery for each channel and the combined significance are shown in Fig. 2 (d). In the collinear mass range $105-140 \mathrm{GeV}$, the combination of the $l l$ and $l h$

\footnotetext{
${ }^{2}$ The estimate on available MC samples has been conservatively multiplied by a factor 5 to reflect our lack of knowledge about this background from real data.
} 


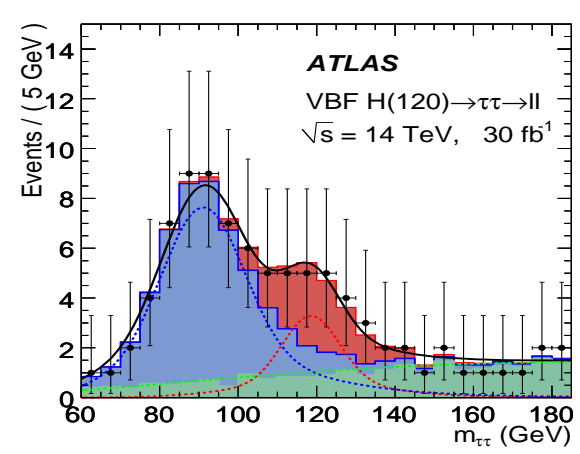

(a)

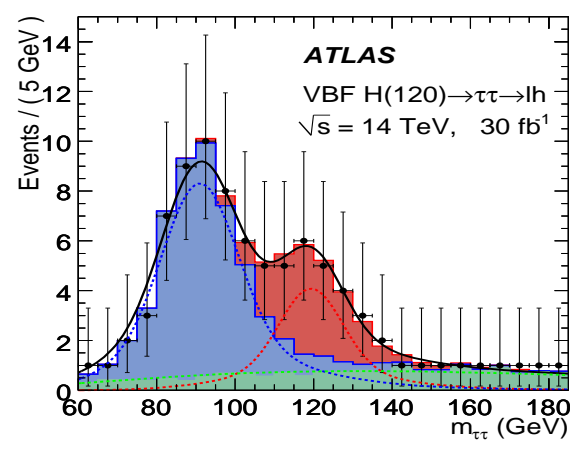

(c)

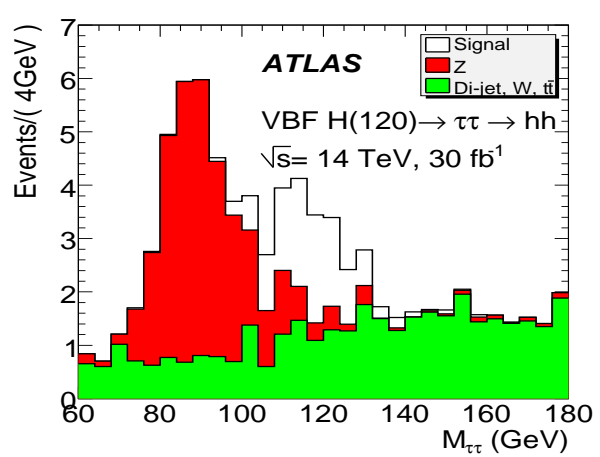

(b)

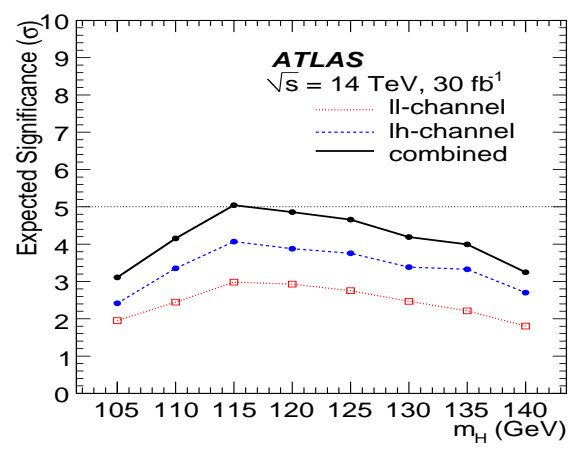

(d)

Figure 2: (a-c): Expected signal and background distributions for the collinear mass for the $l l, l h$ and $h h$ channels at $m_{H}=120 \mathrm{GeV}$, for an integrated luminosity of $30 \mathrm{fb}^{-1}$. Note: in (b) the QCD background component is not mulitplied by the safety factor 5, see text. (d): Expected signal significance for several masses based on fitting the $m_{\tau \tau}$ spectrum. Background uncertainties are incorporated

channel ensures a greater than three sigma observation, with $30 \mathrm{fb}^{-1}\left(3\right.$ years at $\left.10^{33} \mathrm{~cm}^{-2} \mathrm{~s}^{-1}\right)$. The significance is obtained by a Likelihood fit to the number of signal and background events in the collinear mass distribution, in the window defined previously. The results of the fit are superimposed on Fig. $2(\mathrm{a}, \mathrm{c})$. Background uncertainties are incorporated in the fit. It should be noted that this procedure for estimating the significance of an observation of a VBF SM Higgs boson has been made as little MC dependent as possible, therefore it should be rather straight forward to apply it to data. In order to constrain the background rate and shape, we simultaneously fit the signal candidates and the background control samples. In the case of the $l h$-channel, a control sample is the distribution of the number of tracks associated to identified tau leptons decaying hadronically. This allows to constrain the fraction of events without real taus entering the final selection due to misindentification. The background control sample for $Z$ boson plus jets background can be obtained with a signal-free $Z \rightarrow \mu \mu+$ jets sample and transfering the dominant instrumental effects to the $Z \rightarrow \tau \tau+$ jets sample. This is achieved by replacing the muons with an equivalent tau, and carefully treating the decay of the tau. This technique is justified because the $Z \rightarrow \mu \mu+$ jets events have identical jet activity and kinematics as $Z \rightarrow \tau \tau+$ jets (before the tau decays) and because the relevant features of tau decays are well understood. The validity of such procedure has been tested 
on MC samples, and is shown in Fig. 3. These results are obtained without simulaton of pile-up effects in the detector. Some handles against backgrounds, like tau identification and central jet veto, are supposed to be quite sensitive to pile-up effect. Preliminary results show that the current tau identification procedure is robust. Furthermore, a first attempt to estimate the effect of pile-up on the CJV cut is shown in Fig. 3. The effect is visible, but in future studies a veto procedure using track information will be included.
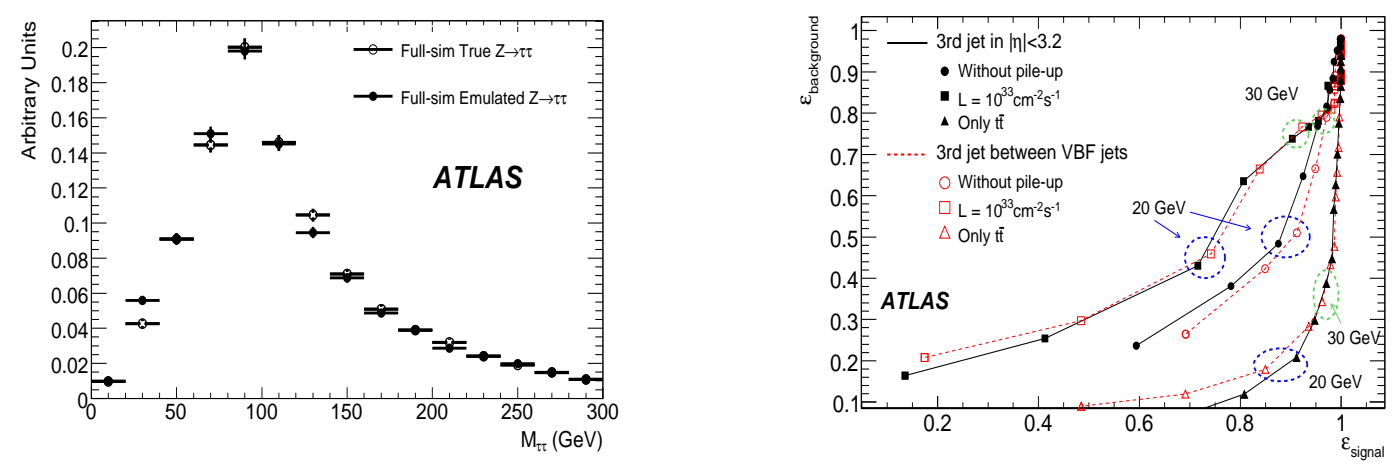

Figure 3: Left: Reconstructed invariant mass distribution generated from the true and emulated $Z \rightarrow \tau \tau \rightarrow$ $l h+3 v$ events. Right: Background rejection versus signal sensitivity for the CJV with and without pileup.

\section{Conclusions}

The sensitivity of the ATLAS detector to a Standard Model Higgs boson produced via vector boson fusion with subsequent decay into taus has been investigated. Particular emphasis has been placed on data-driven background estimation strategies. Results without pileup indicate that more than $\sim 3 \sigma$ significant observation can be achieved in the mass range $105-140 \mathrm{GeV}$ after collecting $30 \mathrm{fb}^{-1}$ of data and combining the $l l$-and $l h$-channels.

\section{References}

[1] ATLAS Collaboration, Expected Performance of the ATLAS Experiment, Detector, Trigger and Physics, CERN-OPEN-2008-020, Geneva, 2008, to appear.

[2] S. Asai et al., "Prospects for the search for a Standard Model Higgs boson in ATLAS using vector boson fusion", Eur. Phys. J. C 32S2 (2004) 19. D. L. Rainwater et al., "Searching for $H \rightarrow \tau \tau$ in weak boson fusion at the LHC", Phys. Rev. D 59 (1999) 014037.

[3] M. Duhrssen et al.,"Extracting Higgs boson couplings from LHC data”, Phys. Rev. D 70 (2004) 113009. M. Duhrssen, ATL-PHYS-2003-030.

[4] T. Plehn, D. L. Rainwater and D. Zeppenfeld, "Determining the structure of Higgs couplings at the LHC”, Phys. Rev. Lett. 88 (2002) 051801. C. Ruwiedel, M. Schumacher, and N. Wermes, "Prospects for the Measurement of the Structure of the Coupling of a Higgs Boson to Weak Gauge Bosons in Weak Boson Fusion with the ATLAS Detector", Eur. Phys. J. C 51 (2007) 385.

[5] T. Plehn, D. L. Rainwater and D. Zeppenfeld, "A method for identifying $H \rightarrow \tau \tau \rightarrow e^{ \pm} \mu^{\mp}$ missing p(T) at the CERN LHC", Phys. Rev. D 61 (2000) 093005. M. Schumacher, "Investigation of the discovery potential for Higgs bosons of the minimal supersymmetric extension of the standard model (MSSM) with ATLAS", hep-ph/0410112. 